\title{
Tunneling through the Quantum Dot Coupled to Incoherent Superconductor
}

\author{
A. Donabidowicz AND T. Domański
}

Institute of Physics, M. Curie Skłodowska University

pl. M. Curie-Skłodowskiej 1, 20-031 Lublin, Poland

Dedicated to Professor Józef Spatek on the occasion of his 60th birthday

We study the equilibrium and non-equilibrium properties of the strongly correlated quantum dot coupled between normal and superconducting leads. The effect of electron pair coherence, Coulomb interactions, and $d$-wave anisotropy of the order parameter are discussed with a particular account of their influence on a charge tunneling through the quantum dot.

PACS numbers: 73.23.-b, 74.20.Fg, 74.45.+c

\section{Introduction}

There have been done numerous theoretical and experimental studies of the quantum dot (QD) at an interface with the superconducting leads [1-6]. Such issue is of a fundamental as well as practical importance and covers a rich area of the physical aspects. It resembles, in principle, the case of a quantum impurity (here understood as some finite system of a discrete energy spectrum) interacting with a sea of the conduction band electrons. Impurities are usually known to have a detrimental effect on the superconducting state (except nonmagnetic impurities weakly interacting with $s$-wave superconductors).

In a configuration of the single electron transistor, where QD serves as a charge and energy transmitter between the external electrodes, this issue becomes even more challenging. If one or both electrodes are superconducting then one needs to establish rather the opposite relation, i.e. of superconductor on the impurity itself. This effect is crucial for the transport properties because the charge and energy currents are very sensitive to energy spectrum of the QD.

According to a general description of the tunneling [7] it has been found that besides the ordinary single electron current there can arise additional contributions from the anomalous channels. They are activated whenever junctions involve the superconducting lead(s) where the particle and hole excitations are mixed with 
each other. For instance, at low biases (smaller than the energy gap) the charge transport occurs only through one of such anomalous Andreev currents [8]. Since it requires electron pairs on the QD, therefore the correlation effects, such as the Coulomb repulsion between electrons on the QD, become extremely important. Indeed, Hekking et al. [9] have shown that the differential conductance of the charge current flowing through the superconducting (S) grain has the characteristic Coulomb blockade oscillations, whose period is $2 e^{2} / 2 C_{\mathrm{G}}$ (where $C_{\mathrm{G}}$ is the gate capacitance) being twice as large as for the normal (N) leads.

Besides the effect of the Coulomb interactions also the fluctuation phenomena between paired electrons have a substantial influence on the transport properties, mainly in the Andreev channel [10]. In the present work we consider a combined effect of the strong correlations together with the electron pair coherence/incoherence focusing on their role for the equilibrium and non-equilibrium properties of N-QD-S junctions. Such analysis would be relevant, when exploring the tunneling, e.g. to the underdoped high $T_{\mathrm{c}}$ superconductors. Main aspects of the present study could also be valid for the scanning tunneling microscopy (STM) measurements.

\section{Microscopic model}

Basic properties of the QD connected to the normal $(\beta=\mathrm{N})$ and superconducting $(\beta=\mathrm{S})$ electrodes can be studied within the impurity-type Hamiltonian

$$
\hat{H}=\hat{H}_{\mathrm{N}}+\hat{H}_{\mathrm{S}}+\sum_{\sigma} \epsilon_{d} \hat{d}_{\sigma}^{\dagger} \hat{d}_{\sigma}+U \hat{n}_{d \uparrow} \hat{n}_{d \downarrow}+\sum_{\boldsymbol{k}, \sigma, \beta}\left(V_{\boldsymbol{k} \beta} \hat{d}_{\sigma}^{\dagger} \hat{c}_{\boldsymbol{k} \sigma \beta}+\text { h.c. }\right) .
$$

Operators $d_{\sigma}\left(d_{\sigma}^{\dagger}\right)$ denote the annihilation (creation) of an electron with spin $\sigma$ and the single particle energy $\varepsilon_{d}$. The on-dot Coulomb repulsion $U>0$ accounts for the charging effects and, under proper conditions, can induce the Kondo resonance at the Fermi level. Mutual interference between the localized electrons of the QD and mobile electrons from the leads is expressed by the hybridization matrix $V_{\boldsymbol{k} \beta}$.

In what follows, we shall treat the normal lead electrons as free fermions $\hat{H}_{\mathrm{N}}=\sum_{\boldsymbol{k}, \sigma}\left(\varepsilon_{\boldsymbol{k N}}-\mu_{\mathrm{N}}\right) \hat{c}_{\boldsymbol{k} \sigma \mathrm{N}}^{\dagger} \hat{c}_{\boldsymbol{k} \sigma \mathrm{N}}$, whose energies belong to a wide band $\left|\varepsilon_{\boldsymbol{k}}\right| \leq D$ such that $\left|V_{k \mathrm{~N}}\right| \ll D$. The other (superconducting) lead will be considered in a general form

$$
\hat{H}_{\mathrm{S}}=\sum_{\boldsymbol{k}, \sigma}\left(\varepsilon_{\boldsymbol{k} S}-\mu_{\mathrm{S}}\right) \hat{c}_{\boldsymbol{k} \sigma \mathrm{S}}^{\dagger} \hat{c}_{\boldsymbol{k} \sigma \mathrm{S}}-\sum_{\boldsymbol{k}, \boldsymbol{k}^{\prime}} U_{\boldsymbol{k}, \boldsymbol{k}^{\prime}} \hat{c}_{\boldsymbol{k} \uparrow \mathrm{S}}^{\dagger} \hat{c}_{-\boldsymbol{k} \downarrow S}^{\dagger} \hat{c}_{-\boldsymbol{k}^{\prime} \downarrow S} \hat{c}_{\boldsymbol{k}^{\prime} \uparrow \mathrm{S}}
$$

The attractive potential $U_{\boldsymbol{k}, \boldsymbol{k}^{\prime}}>0$ is responsible for a formation of the electron pairs. Depending on the temperature $T$ and on magnitude of the pairing potential $U_{\boldsymbol{k}, \boldsymbol{k}^{\prime}}[11,12]$ these pairs can be either coherent (below $T_{\mathrm{c}}$ ) or incoherent (above $T_{\mathrm{c}}$ ). In the high $T_{\mathrm{c}}$ compounds electrons are bound into the local (almost intersite) pairs. It has been recently emphasized by Anderson [13] that in the underdoped region such local pairs do not cease at $T_{\mathrm{c}}$, but still exist at higher temperatures becoming incoherent due to weakening of the phase stiffness. This is in fact not a new idea [11]. Such kind of interpretation has been previously given more than a 
decade ago by Györffy et al. [14] who investigated the incoherent local pairs (ILP) by the selfconsistent coherent potential approximation (CPA) method. We shall use here some of their results which prove to be suitable for the present analysis.

\section{The superconducting state}

For a qualitative description of the superconducting state below $T_{\mathrm{c}}$ one can linearize the pairing interactions reducing the Hamiltonian (2) to the exactly solvable BCS structure

$$
\hat{H}_{\mathrm{S}} \simeq \sum_{\boldsymbol{k}, \sigma}\left(\varepsilon_{\boldsymbol{k} \mathrm{S}}-\mu_{\mathrm{S}}\right) \hat{c}_{\boldsymbol{k} \sigma \mathrm{S}}^{\dagger} \hat{c}_{\boldsymbol{k} \sigma \mathrm{S}}-\sum_{\boldsymbol{k}}\left(\Delta_{\boldsymbol{k}} \hat{c}_{\boldsymbol{k} \uparrow \mathrm{S}}^{\dagger} \hat{c}_{-\boldsymbol{k} \downarrow \mathrm{S}}^{\dagger}+\text { h.c. }\right),
$$

with $\Delta_{\boldsymbol{k}}=\sum_{\boldsymbol{k}^{\prime}} U_{\boldsymbol{k}, \boldsymbol{k}^{\prime}}\left\langle\hat{c}_{-\boldsymbol{k}^{\prime} \backslash S} \hat{c}_{\boldsymbol{k}^{\prime} \uparrow S}\right\rangle$. The appearance of the order parameter $\left\langle\hat{c}_{-\boldsymbol{k} \downarrow S} \hat{c}_{\boldsymbol{k} \uparrow S}\right\rangle \neq 0$ breaks the gauge symmetry of the initial system (2) and consequently leads to a depletion of the single particle states around the Fermi energy $\mu_{\mathrm{S}}$. Instead of single electrons the Cooper pairs take over the role of effective quasiparticles. If the phase stiffness is rigid enough there establishes the off-diagonal long-range order which marks the onset of superconductivity.

To gain some insight into the physics of N-QD-S junction let us first consider the uncorrelated $\mathrm{QD}$, omitting the Coulomb repulsion $U$. For description of the proximity effect it is convenient to introduce the retarded Green function $\boldsymbol{G}_{d}(\omega)=$ $\left\langle\left\langle\hat{\Psi} ; \hat{\Psi}^{\dagger}\right\rangle\right\rangle_{\omega+\mathrm{i} 0^{+}}$in the Nambu representation $\hat{\Psi}^{\dagger}=\left(\hat{d}_{\uparrow}^{\dagger}, \hat{d}_{\downarrow}\right), \hat{\Psi}=\left(\hat{\Psi}^{\dagger}\right)^{\dagger}$. From the Heisenberg equation of motion we obtain

$$
\boldsymbol{G}_{d}^{0}(\omega)^{-1}=\left(\begin{array}{cc}
\omega-\varepsilon_{d} & 0 \\
0 & \omega+\varepsilon_{d}
\end{array}\right)-\boldsymbol{\Sigma}_{d}^{0}(\omega)
$$

where the matrix self-energy is given by

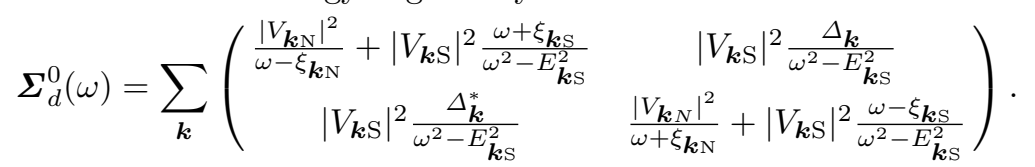

We used a shorthand notation $\xi_{\boldsymbol{k} \beta}=\varepsilon_{\boldsymbol{k} \beta}-\mu_{\beta}$ for energies measured with respect to the chemical potential and $E_{k}=\sqrt{\xi_{k S}^{2}+\left|\Delta_{k}\right|^{2}}$ for the BCS-type quasiparticle dispersion.

The off-diagonal terms of (5) yield a nonvanishing expectation value $\left\langle\hat{d}_{\downarrow} \hat{d}_{\uparrow}\right\rangle \neq 0$. Physically it means that the off-diagonal order of S electrons extends onto the QD. In fact, the excitation spectrum of the QD shows up the characteristic particle-hole features as has been previously pointed out by several authors [6, 10]. We illustrate this in Fig. 1 showing the spectral function $\rho_{d}(\omega)=-\frac{1}{\pi} \operatorname{Im} \boldsymbol{G}_{\mathrm{d}, 11}(\omega)$ for the equilibrium situation $\mu_{\mathrm{N}}=\mu_{\mathrm{S}}$ with the single particle energy $\varepsilon_{d}=\mu_{\beta}$. We clearly notice that the Lorentzian shape (the thin dashed line corresponding to $\mathrm{N}-\mathrm{QD}-\mathrm{N}$ junction) evolves into two peaks (the thick solid line) for the isotropic superconducting lead. Tanaka et al. [6] have recently explained analytically that the distance between such coherence peaks depends merely on $V_{\boldsymbol{k}}$ while their broadening is controlled by $V_{\boldsymbol{k} \mathrm{N}}$. 


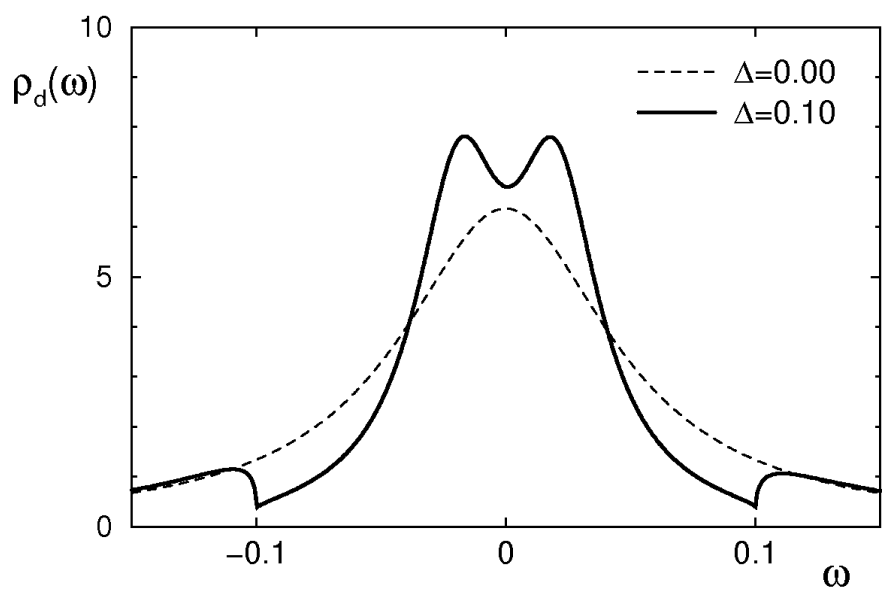

Fig. 1. Spectral function $\rho_{d}(\omega)$ of the noncorrelated QD (for $U=0$ ) in the equilibrium situation, such that $\mu_{\mathrm{N}}=\mu_{\mathrm{S}}=\varepsilon_{d}$. We assumed the flat coupling $\Gamma_{\beta}=$ $2 \pi \sum_{\boldsymbol{k}}\left|V_{\boldsymbol{k} \beta}\right|^{2} \delta\left(\omega-\varepsilon_{\boldsymbol{k} \beta}\right)$, where $\Gamma_{\beta}=0.01 D$ and used $D$ as a unit for energies.

\section{Effect of the phase incoherence}

In electron systems with the strong pairing fluctuations driven by a small concentration of charge carriers or due to partly reduced dimensionality the electron pairs can exist in the normal state over a certain temperature region up to $T^{*}$. The presence of the pairs above $T_{\mathrm{c}}$ is signified by a partial depletion of the low lying energy states in the single particle spectrum. The magnitude and temperature extent of such pseudogap can vary from case to case. In conventional superconductors it is rather marginal [15] while in the high $T_{\mathrm{c}}$ compounds has a pronounced effect [16].

The description of the superconducting fluctuations is a nontrivial issue. Usually it is done perturbatively by including the Maki-Thompson and Aslamazov-Larkin diagrams [17] in the response function. For the strong pair fluctuations such approach is however insufficient, one must go beyond the perturbative scheme. One of convenient methods is feasible in the Lagrangian language. To carry out the integrals over the fermion (Grassmann) fields one can introduce the pairing (boson) field and, via the Hubbard-Stratonovitch transformation, exactly cancel out the quartic term in (2).

Instead of the two-body interactions one is then left with the fermion and boson fields coupled into a bilinear structure. Although there is no direct translation of the effective action back to the Hamiltonian formulation we propose ad hoc the following phenomenological model [11]:

$$
\hat{H}_{\mathrm{S}}=\sum_{\boldsymbol{k} \sigma} \xi_{\boldsymbol{k} \mathrm{S}} \hat{c}_{\boldsymbol{k} \sigma \mathrm{S}}^{\dagger} \hat{c}_{\boldsymbol{k} \sigma \mathrm{S}}+\sum_{j} E_{j}^{b} \hat{b}_{j}^{\dagger} \hat{b}_{j}+g \sum_{\boldsymbol{k}, j}\left(\hat{b}_{j}^{\dagger} \hat{c}_{-\boldsymbol{k} \downarrow \mathrm{S}} \hat{c}_{\boldsymbol{k} \uparrow \mathrm{S}}+\text { h.c. }\right)
$$

accounting for the mixed boson and fermion degrees of freedom. The summation 
for $j$ runs over the spatial sites, where local pairs exist and, for simplicity, we assumed the constant coupling $U_{\boldsymbol{k}, \boldsymbol{k}^{\prime}}=g$. The model (6) is gauge invariant because boson operators describe the objects of a double charge $2 e$.

The two-component model (6) inherits a rich physics generic for the pairing Hamiltonian (2) of the attractive interactions. To focus on the fluctuations of the order parameter we linearize the boson-fermion term $\hat{b}_{j}^{\dagger} \hat{c}_{-\boldsymbol{k} \downarrow S} \hat{c}_{\boldsymbol{k} \uparrow S} \simeq$ $\left\langle\hat{b}_{j}^{\dagger}\right\rangle \hat{c}_{-\boldsymbol{k} \downarrow S} \hat{c}_{\boldsymbol{k} \uparrow S}+\left\langle\hat{c}_{-\boldsymbol{k} \downarrow S} \hat{c}_{\boldsymbol{k} \uparrow S}\right\rangle \hat{b}_{j}^{\dagger}$ thus decoupling the subsystems from one another. The fermion part acquires again the BCS-type structure (3) with a complex order parameter

$$
\Delta_{j}=\left|\Delta_{j}\right| \mathrm{e}^{\mathrm{i} \phi_{j}} \equiv-g\left\langle\hat{b}_{j}\right\rangle .
$$

We next assume the amplitude $\left|\Delta_{j}\right|$ to be uniform and allow only for the phase fluctuations. As recently argued by Anderson [13] this seems to be well justified in the underdoped high $T_{\mathrm{c}}$ materials.

Since above $T_{\mathrm{c}}$ the order parameter does not establish any long-range coherence it is natural to regard the phases $\phi_{j}$ as completely random. Under such circumstances the self-energy (5) can be configurationally averaged with respect to the orientations of $\phi_{j}$ :

$$
\begin{aligned}
& \boldsymbol{\Sigma}_{d, \text { inc }}^{0}(\omega) \simeq\left\langle\boldsymbol{\Sigma}_{d}^{0}(\omega)\right\rangle_{j} \\
& \quad=\sum_{\boldsymbol{k}}\left(\begin{array}{cc}
\frac{\left|V_{\boldsymbol{k}_{\mathrm{N}}}\right|^{2}}{\omega-\xi_{\boldsymbol{k}_{\mathrm{N}}}}+\left|V_{\boldsymbol{k} \mathrm{S}}\right|^{2} \frac{\omega+\xi_{\boldsymbol{k} \mathrm{S}}}{\omega^{2}-E_{\boldsymbol{k} \mathrm{S}}^{2}} & 0 \\
0 & \frac{\left|V_{\boldsymbol{k N}_{\mathrm{N}}}\right|^{2}}{\omega+\xi_{\boldsymbol{k} \mathrm{N}}}+\left|V_{\boldsymbol{k} \mathrm{S}}\right|^{2} \frac{\omega-\xi_{\boldsymbol{k S}}}{\omega^{2}-E_{\boldsymbol{k} \mathrm{S}}^{2}}
\end{array}\right) .
\end{aligned}
$$

The diagonal form of the self-energy (8) does not lead to any splitting of the Lorentzian peak (like in the superconducting state) even though the excitation spectrum is gapped. This is a qualitative difference between the coherent and incoherent pairs as regards their influence on the QD.

To further support the above mentioned finding we briefly consider an alternative way to treat the randomly oriented phases $\phi_{j}$. We use for this purpose the CPA method discussed at length for the negative $U$ Hubbard model [14]. Applying it to the present model (6) one obtains the Green function of S electrons

$$
\boldsymbol{G}_{c}(\boldsymbol{k}, \omega)^{-1}=\left(\begin{array}{cc}
\omega-\xi_{\boldsymbol{k S}} & 0 \\
0 & \omega+\xi_{\boldsymbol{k S}}
\end{array}\right)-\boldsymbol{\Sigma}_{c}(\omega)
$$

with the dynamical, but $\boldsymbol{k}$-independent, self-energy $\boldsymbol{\Sigma}_{c}^{\mathrm{CPA}}(\omega)$. It has been shown that for $T>T_{\mathrm{c}}$ the self-energy becomes diagonal [18] so, in analogy to (5), we can write down the Green function of the QD as

$$
\begin{aligned}
& \boldsymbol{\Sigma}_{d, \text { inc }}^{0}(\omega) \\
& \quad=\sum_{\boldsymbol{k}}\left(\begin{array}{cc}
\frac{\left|V_{\boldsymbol{k}_{\mathrm{N}}}\right|^{2}}{\omega-\xi_{\boldsymbol{k} \mathrm{N}}}+\frac{\left|V_{\boldsymbol{k} \mathrm{S}}\right|^{2}}{\omega-\xi_{\boldsymbol{k} \mathrm{S}}-\Sigma_{c, 11}(\omega)} & 0 \\
0 & \frac{\left|V_{\boldsymbol{k}_{\mathrm{N}}}\right|^{2}}{\omega+\xi_{\boldsymbol{k} \mathrm{N}}}+\frac{\left|V_{\boldsymbol{k}_{\mathrm{S}}}\right|^{2}}{\omega+\xi_{\boldsymbol{k} \mathrm{S}}-\Sigma_{c, 22}(\omega)}
\end{array}\right) .
\end{aligned}
$$

We checked that both Eqs. (8) and (10) give qualitatively identical results. For the 


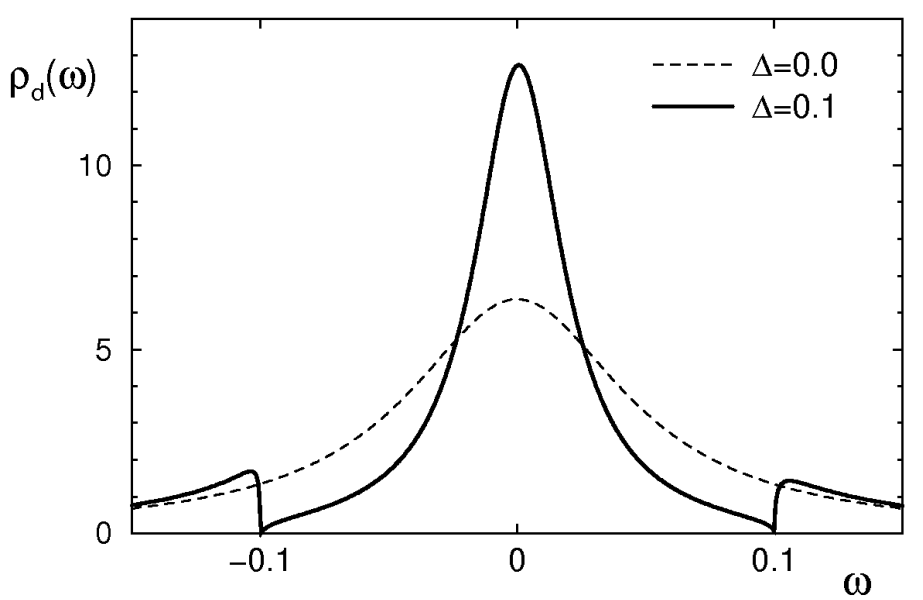

Fig. 2. Spectral function for the same set of parameters as in Fig. 1 but in the pseudogap region above $T_{\mathrm{c}}$ with randomly oriented phases of the order parameter.

transport properties (see Sect. 6) it is very important that above $T_{\mathrm{c}}$ there occurs no proximity effect $\left\langle\hat{d}_{\downarrow} \hat{d}_{\uparrow}\right\rangle=0$. In Fig. 2 we show the resulting spectral function, where only one (partly renormalized) peak is visible instead of two coherent peaks for the superconducting lead (Fig. 1) usually predicted in the theoretical considerations [6].

\section{Influence of the Kondo resonance}

If the Coulomb interactions $U \neq 0$ are taken into account it becomes energetically unfavorable to have an even number of electrons on the QD. Besides such Coulomb blockade there can appear an additional many-body Kondo effect at sufficiently low temperatures [19]. In practical realizations it usually occurs below $1 \mathrm{~K}$ [20], which is by far smaller than the transition temperatures of available superconductors. Therefore, we shall investigate the Kondo state emerging deep in the superconducting region $T_{\mathrm{K}} \ll T_{\mathrm{c}}$.

For the equilibrium or non-equilibrium Kondo effect to appear there must be fulfilled such conditions that the hybridization $V_{\boldsymbol{k} \beta}$ does effectively induce an antiferromagnetic interaction between QD and the leads. In one of possible realizations it happens when the single particle energy level $\varepsilon_{d}$ is located slightly below $\mu_{\beta}$ and simultaneously the Coulomb satellite $U+\varepsilon_{d}$ is safely far above it. This is not a difficult constraint for junctions involving the QD because by varying the gate voltage one can experimentally manipulate the position of $\varepsilon_{d}$.

Skipping unnecessary details we write down the modified Green function for the extreme limit $U \rightarrow \infty$, when the doubly occupied QD states are projected out by means of the slave boson approach taking care of the constraint quantization [5]

$$
\boldsymbol{G}_{d}(\omega)^{-1}=\boldsymbol{G}_{d}^{0}(\omega)^{-1} /\left(1-n_{d \uparrow}\right)-\Sigma^{I}(\omega) .
$$

The additional term $\Sigma^{I}(\omega)$ is explicitly expressed by Eq. (24) in Ref. [5]. For 


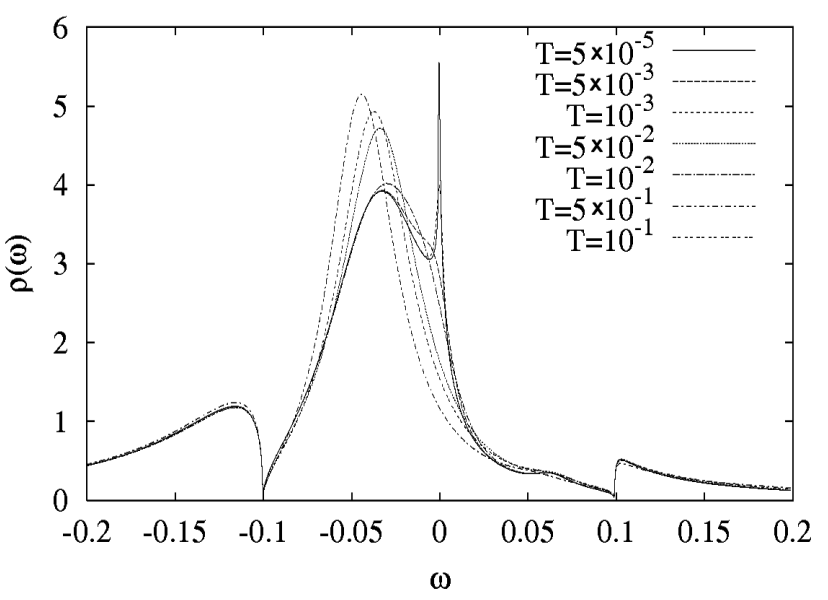

Fig. 3. Spectral function of the strongly correlated QD (for the limit $U=\infty$ ) coupled to a normal lead and isotropic superconductor $\left(T<T_{\mathrm{c}}\right)$. We used the symmetric coupling constants $\Gamma_{\beta}=0.01 D$ and assumed $\varepsilon_{d}$ to be located below the Fermi level within the energy gap of $\mathrm{S}$ electrons $\left|\varepsilon_{d}\right|<\Delta$.

$T \sim T_{\mathrm{K}}$ it gives a narrow resonance at $\mu_{\beta}$. In Fig. 3 we show the spectral function in the equilibrium situation when the single particle energy is located inside the energy gap of superconducting lead $\left|\varepsilon_{d}\right|<\Delta$.

Besides the Kondo peak at $\omega=0^{+}$we notice that the particle-hole features (flat maxima at $\omega \simeq \pm \varepsilon_{d}$ ) are still preserved. The off-diagonal structure of the Green function (11) guarantees that the strongly correlated QD absorbs the off-diagonal ordering of S electrons. On a microscopic level such mechanism of the hybridization induced pairing has been given by Spałek [21] to explain superconductivity of the heavy fermion compounds.

\section{Non-equilibrium transport}

In a presence of the external bias $V$ there are induced the charge and energy flows between the electrodes. To calculate quantitatively the charge current $J(V)=-e \frac{\mathrm{d}}{\mathrm{d} t} \sum_{\boldsymbol{k}, \sigma}\left\langle\hat{c}_{\boldsymbol{k} \mathrm{N} \sigma}^{\dagger} \hat{c}_{\boldsymbol{k N} \sigma}\right\rangle$ it is necessary to work with the non-equilibrium formalism. Using the time evolution equation we find that $J(V)=$ $2 \frac{e}{\hbar} \operatorname{Re}\left\{\sum_{\boldsymbol{k}, \sigma} V_{\boldsymbol{k} \mathrm{N}} G_{d, \boldsymbol{k N}}^{<}\right\}$. The mixed Green function $G_{d, \boldsymbol{k N}}^{<}$can be determined via the Schwinger-Keldysh contour integration. The standard procedure [19] adopted here to the N-QD-S junction gives the following expression for the steady current [5]:

$$
J=J_{11}+J_{12}+J_{22}+J_{A} .
$$

The first term in Eq. (12) describes the single particle tunneling

$$
J_{11}(V)=\frac{2 \mathrm{e} \Gamma_{\mathrm{N}}}{h} \int \mathrm{d} \omega\left(-\operatorname{Im} \Sigma_{11, S}^{r}\left|G_{11}\right|^{2}\right)[f(\omega-e V)-f(\omega)],
$$


where $f(\omega)=\left[1+\exp \left(\omega / k_{\mathrm{B}} T\right)\right]^{-1}$ is the Fermi distribution and we used the constant coupling $\Gamma_{\mathrm{N}}=\sum_{\boldsymbol{k}}\left|V_{\boldsymbol{k N}}\right|^{2} \delta\left(\omega-\varepsilon_{\boldsymbol{k N}}\right)$. When both leads are normal then (13) reduces to the well known Landauer-Büttiker formula [19] $J_{11}(V)=$ $\frac{2 e}{h} \int \mathrm{d} \omega \rho_{d}(\omega) \frac{\Gamma_{\mathrm{N}}(\omega) \Gamma_{\mathrm{S}}(\omega)}{\Gamma_{\mathrm{N}}(\omega)+\Gamma_{\mathrm{S}}(\omega)}[f(\omega-e V)-f(\omega)]$

The other three components in Eq. (12) represent the anomalous channels specific for junctions with the superconducting leads [7]. They can be expressed in the following way [5]:

$$
\begin{aligned}
& J_{12}(V)=\frac{4 e \Gamma_{\mathrm{N}}}{h} \int \mathrm{d} \omega\left(-\operatorname{Im} \Sigma_{12, \mathrm{~S}}^{r} \operatorname{Re}\left[G_{11} G_{12}^{*}\right]\right)[f(\omega-e V)-f(\omega)], \\
& J_{22}(V)=\frac{2 e \Gamma_{\mathrm{N}}}{h} \int \mathrm{d} \omega\left(-\operatorname{Im} \Sigma_{22, \mathrm{~S}}^{r}\left|G_{12}\right|^{2}\right)[f(\omega-e V)-f(\omega)], \\
& J_{A}(V)=\frac{2 e \Gamma_{\mathrm{N}}}{h} \int \mathrm{d} \omega\left(-\operatorname{Im} \Sigma_{22, \mathrm{~N}}^{r}\left|G_{12}\right|^{2}\right)[f(\omega-e V)-f(\omega+e V)] .
\end{aligned}
$$

The last term describes the Andreev current when electron from the $\mathrm{N}$ electrode is transformed into the Cooper pair in superconducting lead with a simultaneous reflection of a hole back into the $\mathrm{N}$ electrode. Such anomalous current enables the charge transfer for the external bias $|\mathrm{eV}| \leq \Delta$.

In Figs. 4 and 5 we show the differential conductance computed numerically for the superconducting (the solid line), pseudogap (the long dashed curve) and normal state (the thin dashed line). Figure 4 describes the situation when $\varepsilon_{d}=0$, so that the applied voltage shifts the chemical potentials to $\mu N=e V / 2, \mu S=$ $-e V / 2$, therefore the conductance becomes symmetric with respect to $V$ (in this case the Kondo peak does not appear). A finite value of $G(V)$ at small voltages

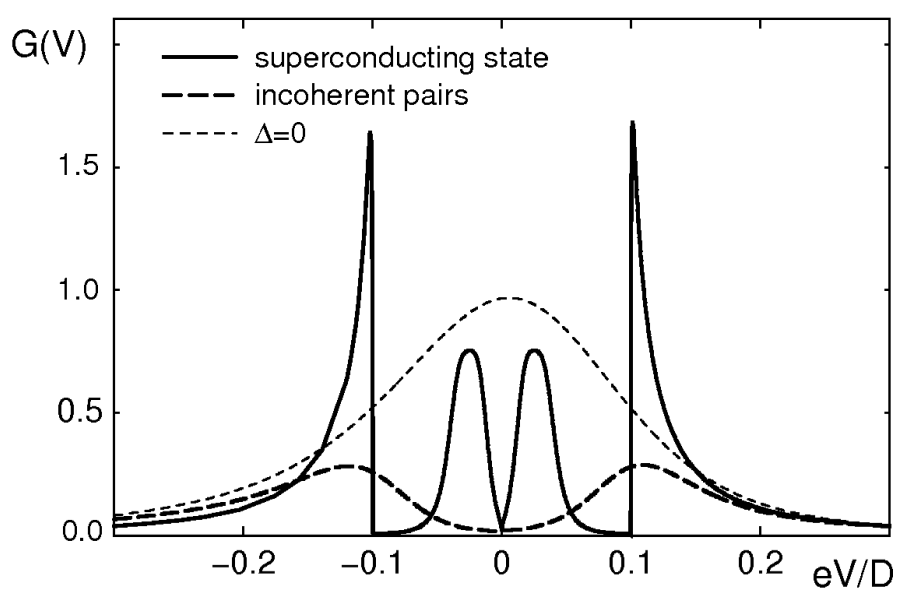

Fig. 4. The differential conductance $G(V)=\mathrm{d} J / \mathrm{d} V$ of $\mathrm{N}-\mathrm{QD}-\mathrm{S}$ junction expressed in the units of $\left.G(0)\right|_{\Delta=0}$. We used $\Delta=0.1 D$ and $\varepsilon_{d}=0$. The solid line corresponds to the coherent electron pairs $\left(T<T_{\mathrm{c}}\right)$ while the dashed curve describes the incoherent pairs (above $T_{\mathrm{c}}$ ). 


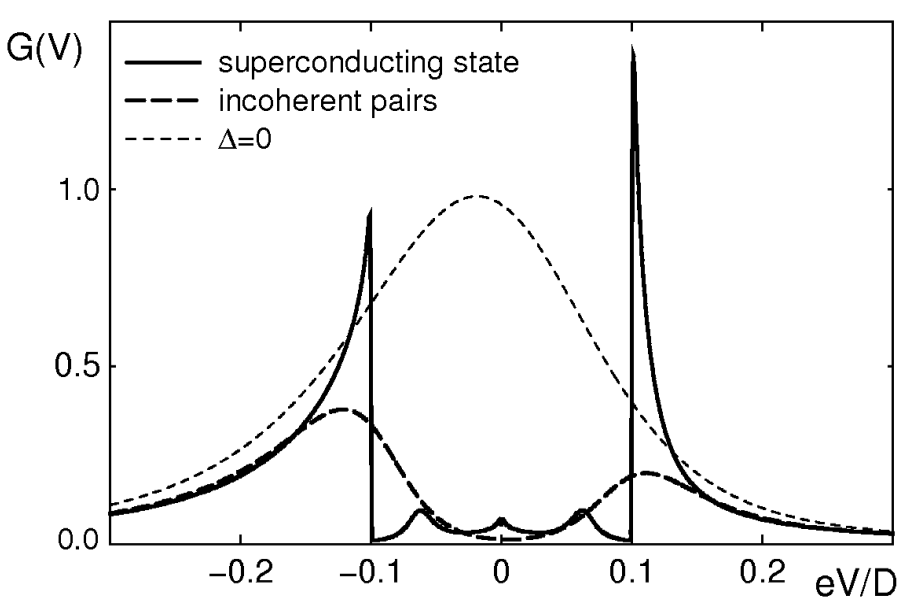

Fig. 5. The same as in Fig. 4 but for a different single energy level $\varepsilon_{d}=-0.08 D$ in the Kondo regime (see Fig. 3). Let us note a residual zero bias enhancement due to the effect of the Kondo peak on the Andreev current.

$|e V|<\Delta$ is due to the anomalous Andreev current. There can be noted two maxima of such Andreev current coinciding with the coherence peaks of the QD spectrum shown in Fig. 1. In the pseudogap region these peaks are absent hence there is no maximum inside the pseudogap region.

If the single particle level is located below $\mu_{\beta}$ then the Kondo peak builds up and it gives a tiny enhancement of the zero bias conductance. Obviously it is contributed by the Andreev current. Such enhanced conductance is much smaller than the unitary limit value $2 e^{2} / h$ predicted and observed in the $\mathrm{N}-\mathrm{QD}-\mathrm{N}$ junction [20].

\section{Effect of $d$-wave anisotropy}

In the high temperature superconductors pairing occurs in the quasi-two-dimensional $\mathrm{CuO}_{2}$ planes, practically between electrons on neighboring lattice sites. The underdoped samples are there characterized by a weak phase stiffness because of a close neighborhood to the insulating state as well as competition with other kinds of the local order (magnetic stripes, charge density waves, etc.). Upon increasing the temperature to $T_{\mathrm{c}}$ electron pairs become incoherent rather than dissociate into the single species. In this section we give an account of the other important property, namely the $d$-wave anisotropy of the order parameter $\Delta_{k}=\Delta\left[\cos k_{x}-\cos k_{y}\right]$. It reflects the fourfold symmetry of $\mathrm{CuO}_{2}$ planes and in consequence gives the gapless ( $V$-shape) energy spectrum of the high $T_{\mathrm{c}}$ superconductors.

Due to the symmetry reasons the Green function of the QD coupled to the $d$-wave superconductor cannot have any off-diagonal terms. Independently of the Coulomb interactions the matrix self-energy $\Sigma_{d}(\omega)$ becomes diagonal after 
integrating over momentum $\boldsymbol{k}=\left(k_{x}, k_{y}\right)$ since $\Delta_{\boldsymbol{k}}$ varies its sign. In a way this situation resembles the pseudogap case discussed in Sect. 4 .

Figure 6 shows the spectral function $\rho_{d}(\omega)$ for the equilibrium case. Coherence peaks of the $d$-wave superconductor (see the inset) are here not divergent like the square root singularities of the isotropic superconductor therefore the kinks of QD spectrum at $\omega= \pm \Delta(T)$ become quite shallow. The Lorentzian peak is a bit deformed in the present case due to $\omega$-dependence of the imaginary part $\operatorname{Im} \Sigma_{d}(\omega)$ which is responsible for a width. We also clearly notice a substantial reduction of the Kondo peak because of the partly depleted states in the superconductor for $|\omega| \leq \max \left\{\Delta_{k}\right\}$.

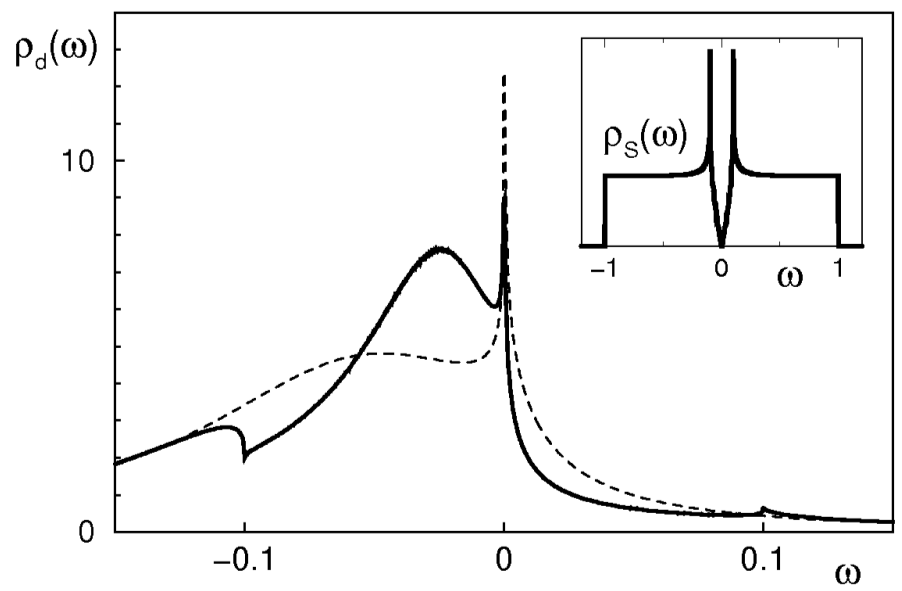

Fig. 6. Spectral function $\rho_{d}(\omega)$ of the quantum dot (in the limit $U=\infty$ ) coupled to a normal lead and $d$-wave superconductor. The thin dashed line corresponds to $\Delta=0$ while the thick solid curve to $\Delta=0.1 D$. We used $\varepsilon_{d}=-0.08 D, \Gamma_{\beta}=0.01 D$, $\Delta(T)=0.1 D$, and temperature $T<T_{\mathrm{K}} \ll T_{\mathrm{c}}$. The inset shows the density of states of the $d$-wave superconducting electrode.

Nevertheless, it has been pointed out by Borkowski and Hirschfeld [22] and more recently by other authors [23] that gapless superconductivity does not preclude appearance of the Kondo resonance. We show in Fig. 6 such Kondo resonance obtained at low temperatures for the QD symmetrically coupled to the $d$-wave superconductor. However, without the off-diagonal terms in $\boldsymbol{G}_{d}(\omega)$ there is no contribution of the anomalous channels to the tunneling. One may thus ask whether the Kondo peak is going to enhance the differential conductance in very much the same way as it does when both leads are normal [20] or one of them is the isotropic superconductor [10]. There is no simple answer to this question [24]. We shall discuss it separately using the sophisticated methods to account for feedback of the Kondo state on energy spectrum of the $d$-wave superconducting lead. 


\section{Conclusions}

We studied the thermodynamic and transport properties of the quantum dot coupled to normal and superconducting electrodes. We show that the isotropic superconductor induces a proximity effect in the QD. It does no longer occur above $T_{\mathrm{c}}$ when electron pairs lose their long-range coherence, even though the energy gap is preserved. Measurements of the non-equilibrium charge current through the quantum dot could thus be a sensitive tool for probing the superconducting fluctuations. For the superconducting state below $T_{\mathrm{c}}$ we find evidence for a marginal zero bias anomaly of the differential conductance due to the Kondo effect. Further studies are needed for the $d$-wave superconductors where, due to the symmetry reasons, the proximity effect is inefficient. Eventual resonant states inside the $V$ shaped gap [24] might have nontrivial effects distinguishing the regions of coherent from incoherent electrons pairs.

\section{Acknowledgments}

We acknowledge a partial support by the State Committee for Scientific Research under the grant No. 2P03B06225.

\section{References}

[1] R. Fazio, R. Raimondi, Phys. Rev. Lett. 80, 2913 (1998); Phys. Rev. Lett. 82, 4950 (1998); P. Schwab, R. Raimondi, Phys. Rev. B 59, 1637 (1999).

[2] Q.-F. Sun, J. Wang, T.-H. Lin, Phys. Rev. B 59, 3831 (1999).

[3] A.A. Clerk, A. Ambegaokar, S. Herschfield, Phys. Rev. B 61, 3555 (2000).

[4] J.C. Cuevas, A. Levi Yeyati, A. Martin-Rodero, Phys. Rev. B 63, 094515 (2001).

[5] M. Krawiec, K.I. Wysokiński, Supercond. Sci. Technol. 17, 103 (2004).

[6] Y. Tanaka, N. Kawakami, A. Oguri, cond-mat/0701570.

[7] G.E. Blonder, M. Tinkham, T.M. Klapwijk, Phys. Rev. B 25, 4515 (1982).

[8] G. Deutscher, D. Feinberg, Appl. Phys. Lett. 76, 486 (2000).

[9] F.W. Hekking, L.I. Glazman, K.A. Matveev, R.I. Shekhter, Phys. Rev. Lett. 70, 4138 (1993).

[10] T. Domański, A. Donabidowicz, K.I. Wysokiński, cond-mat/0612440.

[11] R. Micnas, J. Ranninger, S. Robaszkiewicz, Rev. Mod. Phys. 62, 113 (1990).

[12] M. Randeria, in: Bose Einstein Condensation, Eds. A. Griffin, D. Snoke, S. Stringari, Cambridge Univ. Press, Cambridge 1995, p. 355.

[13] P.W. Anderson, cond-mat/0701042.

[14] B.L. Györffy, J.B. Stauton, G.M. Stocks, Phys. Rev. B 44, 5190 (1991).

[15] R.W. Cohen, B. Abeles, C.R. Fuselier, Phys. Rev. Lett. 23, 377 (1969).

[16] P.A. Lee, N. Nagaosa, X.-G. Wen, Rev. Mod. Phys. 78, 17 (2006).

[17] A.A. Varlamov, G. Balestrino, E. Milani, D.V. Livanov, Adv. Phys. 48, 655 (1999). 
[18] P. Curty, H. Beck, Phys. Rev. Lett. 91, 257 (2003).

[19] Y. Meir, N.S. Wingreen, Phys. Rev. Lett. 67, 2512 (1992); Y. Meir, N.S. Wingreen, P.A. Lee, Phys. Rev. Lett. 70, 2601 (1993).

[20] D. Goldhaber-Gordon, H. Shtrikman, D. Mahalu, D. Abusch-Magder, U. Mairav, M.A. Kastner, Nature 391, 156 (1998).

[21] J. Spałek, Phys. Rev. B 38, 208 (1988).

[22] L.S. Borkowski, P.J. Hirschfeld, Phys. Rev. B 46, 9274 (1992).

[23] M. Vojta, R. Bulla, Phys. Rev. B 65, 014511 (2002); A. Polkovnikov, S. Sachdev, M. Vojta, Phys. Rev. Lett. 86, 296 (2001).

[24] R. Bulla, T. Costi, Th. Pruschke, cond-mat/0701105. 